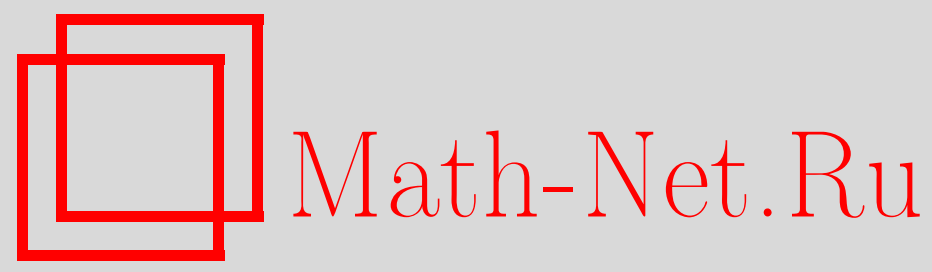

А. В. Пухликов, Послойные бирациональные соответствия, Матем. заметки, 2000, том 68, выпуск 1, 120-130

DOI: https://doi.org/10.4213/mzm926

Использование Общероссийского математического портала Math-Net.Ru подразумевает, что вы прочитали и согласны с пользовательским соглашением http://www. mathnet.ru/rus/agreement

Параметры загрузки:

IP : 18.234 .197 .8

26 апреля 2023 г., 14:01:30

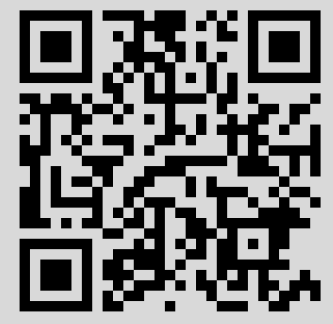




\title{
ПОСЛОЙНЫЕ БИРАЦИОНАЛЬНЫЕ СООТВЕТСТВИЯ
}

\author{
А. В. Пухликов
}

Доказано, что послойное бирациональное соответствие между гладкими расслоениями на гиперповерхности Фано над кривой, индуцирующее (бирегулярньй) изоморфизм общего слоя, есть послойный изоморфизм.

Библиография: 12 названий.

1. Введение. Если $V, V^{\prime}$ - алгебраические многообразия одинаковой размерности над одним и тем же полем констант $k$, то либо множество $\operatorname{Bir}_{k}\left(V, V^{\prime}\right)=\left\{\chi: V \rightarrow V^{\prime}\right\}$ бирациональных соответствий пусто, либо для любого $\chi \in \operatorname{Bir}_{k}\left(V, V^{\prime}\right)$ композиция

$$
\circ \chi: \operatorname{Bir}_{k} V \rightarrow \operatorname{Bir}_{k}\left(V, V^{\prime}\right), \quad \circ \chi: \varphi \mapsto \chi \circ \varphi: V \rightarrow V^{\prime}
$$

определяет биекцию группы бирациональных автоморфизмов многообразия $V$ над $k$ и множества $\operatorname{Bir}_{k}\left(V, V^{\prime}\right)$. Таким образом, задача бирациональной классификации алгебраических многообразий (возможно, ограниченная рамками определенного класса многообразий) распадается на две: во-первых, необходимо уметь распознавать бирационально эквивалентные многообразия, т.е. отвечать на вопрос о существовании бирационального отображения $V \rightarrow V^{\prime}$ для заданных $V, V^{\prime}$, и, во-вторьх, вьгислять группу бирациональных автоморфизмов $\operatorname{Bir}_{k} V$ заданного многообразия. Наиболее нетривиальньми и содержательными задачи бирациональной классификации являются для расслоений Фано $\pi: V \rightarrow S$, общий слой которых $V_{s}=\pi^{-1}(s), s \in S$, есть многообразие Фано.

Для бирационального отображения $\chi: V \rightarrow V^{\prime}$ между расслоениями $\Phi$ ано $V / S$ и $V^{\prime} / S^{\prime}$ первьй и ключевой вопрос состоит в том, согласовано ли $\chi$ со структурами расслоений $Ф$ ано. Иначе говоря, достраивается ли диаграмма

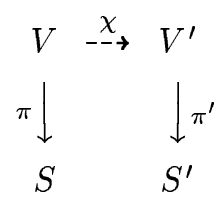

до коммутативной посредством подходящего (рационального доминантного) отображения $\alpha: S \rightarrow S^{\prime}$ ? В последние десятилетия было понято, что в некотором смысле для

Работа выполнена при поддержке Российского фонда фундаментальных исследований, гранты № 99-15-96013 и № 99-01-01132, и грантом INTAS-OPEN 97/2072. 
"подавляющего большинства" расслоений Фано $V / S$ ответ оказьвается положительным при произвольных $V^{\prime} / S^{\prime}$ и $\chi$. Это так назьваемьй феномен бирациональной жсест$\kappa о с т и$, угаданньй $Ф$ ано сто лет назад и на строгой основе открытьй В. А. Исковских и Ю.И. Маниным в [1] $\left(V=V_{4} \subset \mathbb{P}^{4}\right.$ - гладкая трехмерная квартика, $S=*-$ точка). Позднее было обнаружено много классов бирационально жестких расслоений Фано (см. [2]-[6]), что и позволило сделать вьвод о типичности этого свойства.

Для бирационально жесткого расслоения $\Phi$ ано $V / S$ задачи бирациональной классификации сводятся к послойной бирациональной классификации. Точная последовательность

$$
1 \rightarrow \operatorname{Bir}(V / S) \rightarrow \operatorname{Bir} V \rightarrow \operatorname{Bir} S
$$

сводит вычисление группы бирациональных автоморфизмов к вычислению группы собственных бирациональных автоморфизмов, сохраняющих слои $\pi$ :

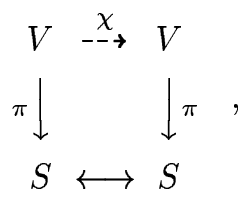

или, что то же самое, групшы $\operatorname{Bir} F_{\eta}$ бирациональных автоморфизмов слоя $F_{\eta}$ над общей (незамкнутой) точкой базы $S$. Аналогично, для бирационально жесткого расслоения $\Phi$ ано $V / S$ вопрос о существовании бирационального отображения $V \rightarrow-V^{\prime} / S^{\prime}$ решается в рамках послойных бирациональных соответствий.

Рассмотрим послойное бирациональное соответствие

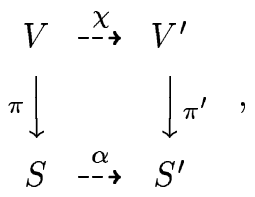

где $\alpha \in \operatorname{Bir}\left(S, S^{\prime}\right)$ - бирациональное соответствие. Отображение $\chi$ индуцирует бирациональное соответствие на общих слоях $\chi: F_{\eta} \rightarrow F_{\eta^{\prime}}^{\prime}$. Можно понимать $\chi$ и как непрерьвное семейство бирациональных отображений слоев $S \ni s \mapsto \chi_{s} \in \operatorname{Bir}\left(F_{s}, F_{\alpha(s)}^{\prime}\right)$. Предположим теперь, что слой $F_{s}$ общего положения есть бирационально сверхжесткое [4] многообразие. Тогда бирациональные эквивалентности слоев $\chi_{s}$ суть бирегулярные изоморфизмы, и мы получаем задачу описания послойных бирациональных отображений $\chi$, индуцирующих бирегулярньй изоморфизм на слое общего положения.

Эта задача локальна по базе $S$ расслоения Фано и заключается в описании возможных перестроек специальных слоев.

2. Основной результат. В настоящей работе изучаются послойные бирациональные соответствия гладких расслоений $Ф$ ано над кривой, слой которых есть гиперповерхность Пусть $C$ - гладкая алгебраическая кривая (вообще говоря, аффинная кривая) с отмеченной точкой $p \in C$ и $C^{*}=C \backslash\{p\}$ - "проколотая" кривая. Все построения остаются верными, если вместо $C$ рассматривать “росток гладкой кривой" в точке $p$ или малый диск $\Delta_{\varepsilon}=\{|z|<\varepsilon\} \subset \mathbb{C}$. Символ $\mathbb{P}$ обозначает комплексноепроективное пространство $\mathbb{P}^{M}, M \geqslant 3$. Пусть $\mathscr{V}(d)$ - класс гладких дивизоров $V \subset X=C \times \mathbb{P}$, слой которых 
$F_{x}=V \cap X_{x}, X_{x}=\{x\} \times \mathbb{P}, x \in C$, есть гиперповерхность степени $d \geqslant 2$. Положим $X^{*}=C^{*} \times \mathbb{P}, V^{*}=V \cap X^{*}$, так что $V^{*}$ получается из $V$ удалением слоя $F_{p}$ над отмеченной точкой. Основной результат работы

Теорема 1. Предполохсим, что $d \geqslant 3$. Пусть $V_{1}, V_{2} \in \mathscr{V}(d) u \chi^{*}: V_{1}^{*} \rightarrow V_{2}^{*}-$ послойный изоморфизм. Тогда $\chi^{*}$ продолжсается до послойного изоморфизма $\chi: V_{1} \rightarrow V_{2}$.

Иными словами, в пределах класса $\mathscr{V}(d)$ многообразия не допускают нетривиальных бирациональных перестроек слоев.

Кроме того, будут описаны бирациональные перестройки расслоений на квадрики $(d=2)$ и самого расслоения $X$.

Пусть $\mathbb{Z}_{\geqslant 2}$ обозначает множество целых чисел $m \geqslant 2$.

ГИПотеЗА. Для заданного $k \geqslant 2$ существуют иелое $M_{*} \geqslant k+2$ и конечное подмнохсество $S \subset \mathbb{Z}_{\geqslant 2}^{k}$ (возмохнно пустое) такие, что для любого $M \geqslant M_{*}$ и любого набора $\left(d_{1}, \ldots, d_{k}\right) \in \mathbb{Z}_{\geqslant 2}^{k} \backslash S$ утвержсдение теоремы 1 справедливо для класса $\mathscr{V}\left(d_{1}, \ldots, d_{k}\right)$ гладких полных пересечений типа $\left(d_{1}, \ldots, d_{k}\right)$ в $C \times \mathbb{P}^{M}$.

Другое направление обобщения теоремы 1 связано с рассмотрением более широкого класса дивизоров в $C \times \mathbb{P}$, допускающего особенности на $V$, а не только на слоях $V \rightarrow C$. Нет сомнений, что для “достаточно мягких" особенностей теорема 1 остается верной. Весьма интересной является задача отыскания границы, после перехода через которую возникают нетривиальные перестройки слоев.

До сих пор задачам послойных перестроек уделялось мало внимания [7], и там, где они рассматривались, они играли вспомогательную роль (так, перестройки расслоений на коники использовались в [2] для доказательства бирациональной жесткости). По сути, задача считалась решенной, если бирациональное отображение расслоений $Ф а-$ но оказывалось послойным. И действительно, "послойные" задачи несравнимо легче, чем “канонические” задачи бирациональной геометрии [1]-[5]. Тем не менее, послойные отображения заслуживают внимания. Первая работа в этом направлении была сделана И. Паком [8], доказавшим отсутствие перестроек гладких слоев для гладких трехмерных многообразий с пучком поверхностей дель Пецио степени $\leqslant 4$. За ней последовала работа [9], в которой доказано отсутствие перестроек гладких слоев для 4-мерных многообразий с пучком трехмерных квартик. Кроме того, И. А. Чельцов анонсировал аналогичньй результат для пучков гиперповерхностей $\Phi$ ано $W_{M} \subset \mathbb{P}^{M}$ произвольной размерности.

Рассуждения Чельцова и Пака основаны на результатах программы лог-минимальных моделей, главньм образом на теореме В. В. Шокурова о связности [10], [11]. Между тем, как показьвает наше доказательство, теорема 1 - это простой факт проективной геометрии, не требующий лог-пар и связанной с ними техники. Наше рассуждение совершенно элементарно и дает более сильное утверждение, чем теоремы Чельцова и Пака, потому что мы не требуем, чтобы специальный слой над отмеченной точкой был неособым: достаточно гладкости многообразия $V$.

В частности, теорема 1 влечет следующий факт.

СлЕДСТВИЕ. Пусть $V / \mathbb{P}^{1} u V^{\prime} / \mathbb{P}^{1}$ - гладкие расслоения на гиперповерхности Фано индекса 1 и $\mathrm{V} / \mathbb{P}^{1}$ достаточно закручено по базе [5]. Тогда любое бирациональное отображсние $\chi: V \rightarrow V^{\prime}$ есть послойный бирегулярный изоморфизм. 
Настоящая работа является естественным дополнением [5] и в значительной степени продолжает мою работу с многомерными расслоениями Фано, которую я проводил в Математическом институте Макса Планка в 1997 году в Бонне.

3. Расслоения на полные пересечения. Пусть $\left(d_{1}, \ldots, d_{k}\right) \in \mathbb{Z}_{\geqslant 2}^{k}$ фиксированньй тип полного пересечения. Рассмотрим класс подмногообразий в $C \times \mathbb{P}$, локально по $C$ имеющих представление $f_{1}=\cdots=f_{k}=0$, где относительно системы $\left(x_{0}: \cdots: x_{M}\right)$ однородных координат на $\mathbb{P}$ уравнения $f_{i}$ имеют вид

$$
f_{i}=\sum_{|I|=d_{i}} a_{I} x^{I},
$$

$I=\left(j_{0}, \ldots, j_{M}\right)$ - мультииндексы степени $j_{0}+\cdots+j_{M}=d_{i}$ и коэффициенты $a_{I}$ суть регулярные функции на $C$, причем для любой точки $y \in C$ набор уравнений $\left\{f_{*}\right\}$, ограниченньй на слой $X_{y}=\{y\} \times \mathbb{P} \cong \mathbb{P}$, задает полное пересечение коразмерности $k$ в $\mathbb{P}$. Обозначим класс таких многообразий через $\mathscr{Z}\left(d_{1}, \ldots, d_{k}\right)$. Пусть $V \in \mathscr{Z}\left(d_{1}, \ldots, d_{k}\right)$ и $F=V \cap X_{p}-$ слой над отмеченной точкой.

Зафиксируем систему уравнений $\left\{f_{*}\right\}$ для $V$ около точки $p \in C$ и локальньй параметр $t$ на кривой $C$ в точке $p$. Теперь уравнения $f_{i}$ можно разложить в ряд Тейлора $f_{i}=f_{i}^{(0)}+t f_{i}^{(1)}+\cdots+t^{j} f_{i}^{(j)}+\cdots$, где $f_{i}^{(j)}$ - однородные многочлены степени $d_{i}$ от $\left(x_{*}\right)$. Слой $F \subset \mathbb{P}$ задается системой уравнений $\left\{f_{*}^{(0)}=0\right\}$.

Лемма 1. Имеет место оценка $\operatorname{dim}\left(X_{p} \cap \operatorname{Sing} V\right) \geqslant \operatorname{dim} \operatorname{Sing} F-1$.

ДокАЗАТЕльство аналогично доказательству леммы 3.4.2 в [12]. Множество $\operatorname{Sing} F$ задается на $F$ условием $\mathrm{rk}\left\|\partial f_{i}^{(0)} / \partial x_{j}\right\| \leqslant k-1$. Если $\operatorname{dim} \operatorname{Sing} F \leqslant 0$, то доказывать нечего. В противном случае пусть $Y \subset \operatorname{Sing} F$ - компонента максимальной размерности, $\operatorname{dim} Y \geqslant 1$. Множество $X_{p} \cap \operatorname{Sing} V$ задается на $F$ условием

$$
\mathrm{rk}\left\|\frac{\partial f_{i}^{(0)}}{\partial x_{j}} \mid f_{i}^{(1)}\right\| \leqslant k-1 .
$$

Если множество $D=\left\{x \in Y \mid \mathrm{rk}\left\|\partial f_{i}^{(0)} / \partial x_{j}\right\| \leqslant k-2\right\}$ имеет в $Y$ коразмерность 1, то лемма доказана, так как $D \subset X_{p} \cap \operatorname{Sing} V$. Предположим противное: $\operatorname{codim}_{Y} D \geqslant 2$. Возьмем общую кривую $\Gamma \subset Y$, не имеющую общих точек с $D$. Во всех точках кривой $\Gamma$ ранг матрицы $\left\|\partial f_{i}^{(0)} / \partial x_{j}\right\|$ постоянен и равен $k-1$. Рассмотрим морфизмы пучков

$$
\mu_{j}: \bigoplus_{i=1}^{k} \mathscr{O}_{\Gamma}\left(1-d_{i}\right) \rightarrow \mathscr{O}_{\Gamma},
$$

заданные локально на наборах сечений $\left(s_{1}, \ldots, s_{k}\right)$ формулой

$$
\mu_{j}:\left(s_{1}, \ldots, s_{k}\right) \mapsto \sum_{i=1}^{k} s_{i} \frac{\partial f_{0}^{(i)}}{\partial x_{j}}
$$

относительно фиксированного отождествления $\mathscr{O}(-a) \otimes \mathscr{O}(a) \cong \mathscr{O}$. По предположению подпучок

$$
\operatorname{Ker}\left(\mu_{*}\right)=\bigcap_{j=0}^{M} \operatorname{Ker} \mu_{j} \subset \bigoplus_{i=1}^{k} \mathscr{O}_{\Gamma}\left(1-d_{i}\right)
$$


имеет постоянный ранг 1. Рассмотрим морфизм пучков

$$
\lambda: \operatorname{Ker}\left(\mu_{*}\right) \rightarrow \mathscr{O}_{\Gamma}(1), \quad \lambda:\left(s_{1}, \ldots, s_{k}\right) \mapsto \sum_{i=1}^{k} s_{i} f_{i}^{(1)}
$$

Предположим, что условие (1) не выполнено ни в одной точке кривой $Г$. Тогда $\lambda$ - изоморфизм обратимьх пучков, т.е.

$$
\mathscr{O}_{\Gamma}(1) \hookrightarrow \bigoplus_{i=1}^{k} \mathscr{O}_{\Gamma}\left(1-d_{i}\right)
$$

что невозможно.

Обратимся к расслоениям на гиперповерхности. Согласно лемме 1 многообразие $V \in \mathscr{Z}(d)$ с локальным уравнением $f=f^{(0)}+t f^{(1)}+\cdots$ является гладким, т.е. $V \in \mathscr{V}(d)$, в том и только том случае, если выполнены два условия:

i) гиперповерхность $F=\left\{f^{(0)}=0\right\}$ имеет не более чем нульмерные особенности;

ii) для любой точки $x \in \operatorname{Sing} F$ имеем $f^{(1)}(x) \neq 0$.

4. Диагональное представление. Пусть $V_{1}, V_{2} \in \mathscr{V}(d), d \geqslant 2$, и $\chi^{*}: V_{1}^{*} \rightarrow V_{2}^{*}-$ послойный изоморфизм вне отмеченной точки $p \in C$. Поскольку слои над точками обшего положения $y \in C$ суть гладкие гиперповерхности степени $d \geqslant 2$, над точкой $y \in C^{*}$ изоморфизм $\chi_{y}^{*}$ индуцирован автоморфизмом объемлющего проективного пространства $\xi_{y} \in$ Aut $\mathbb{P}$. Таким образом, $\chi^{*}=\left.\xi^{*}\right|_{V_{1}}$, где $\xi_{y}^{*}=\xi_{y}$ есть алгебраическая кривая $\xi^{*}: C^{*} \rightarrow$ Aut $\mathbb{P}$ проективных автоморфизмов. Пусть $\mathbb{P}=\mathbb{P}(L)$, где $L \cong \mathbb{C}^{M+1}$ линейное пространство. Кривая $\xi^{*}$ может быть поднята до кривой $\xi: C \rightarrow \operatorname{End} L$, $\xi\left(C^{*}\right) \subset$ Aut $L$. Если $\xi(p) \in$ Aut $L$, то $\chi^{*}$ продолжается до послойного (бирегулярного) изоморфизма $\chi=\left.\xi\right|_{V_{1}}$ и многообразия $V_{1}$ и $V_{2}$ послойно изоморфны. Предположим, что это не так: $\operatorname{det} \xi(p)=0$.

Зафиксируем локальньй параметр $t$ на кривой $C$ в точке $p$, и пусть $\sum_{i=0}^{\infty} t^{i} \xi^{(i)}-$ ряд Тейлора кривой $\xi$. Можно считать, что $\xi^{(0)} \neq 0$.

Лемма 2. Существуют кривые әндоморфизмов $\beta, \gamma: C \rightarrow$ End $L$ и базис $\left(e_{0}, \ldots\right.$, $\left.e_{M}\right)$ пространства $L$ такие, что $\beta(p), \gamma(p) \in$ Aut $L, u$ в этом базисе кривая $\beta \xi \gamma^{-1}: C \rightarrow$ End $L$ имеет диагональный вид:

$$
\beta \xi \gamma^{-1}: e_{i} \mapsto t^{w\left(e_{i}\right)} e_{i}
$$

əде $w\left(e_{i}\right) \in \mathbb{Z}_{+}$.

ДокАЗАТЕЛЬСтво. Это хорошо известный факт Элементарной линейной алгебры.

Заменим $V_{1}$ на $\gamma\left(V_{1}\right)$ и $V_{2}$ на $\beta\left(V_{2}\right)$. Теперь мы можем просто считать, что послойное бирациональное соответствие $\xi$ имеет вид (2) с самого начала. Покажем, что если $m=\max \left\{w\left(e_{i}\right)\right\} \geqslant 1$, то этого не может быть.

Пусть $\left\{a_{0}=0<a_{1}<\cdots<a_{k}\right\}=\left\{w\left(e_{i}\right), i=0, \ldots, M\right\} \subset \mathbb{Z}_{+}-$множество весов диагонального преобразования $(2), k \leqslant M, m=a_{k}$ - максимальньй вес. Рассмотрим 
систему однородных координат $\left(x_{0}: \cdots: x_{M}\right)$, двойственную базису $\left(e_{*}\right)$. Определим вес мономов от $x_{*}$, полагая

$$
w\left(x_{0}^{n_{0}} x_{1}^{n_{1}} \cdots x_{M}^{n_{M}}\right)=\sum_{i=0}^{M} n_{i} w\left(e_{i}\right)
$$

Положим $\mathscr{A}_{i}=\left\{x_{j} \mid w\left(e_{j}\right)=a_{i}\right\} \subset \mathscr{A}=\left\{x_{0}, \ldots, x_{M}\right\}$ - множество координат веса $a_{i}$. Выделим особо множества $\mathscr{A}_{*}=\mathscr{A}_{0}$ и $\mathscr{A}^{*}=\mathscr{A}_{k}$ координат минимального и максимального веса соответственно.

5. Доказательство теоремы 1. Пусть $f=f^{(0)}(x)+t f^{(1)}+\cdots$ - локальное по базе $C$ уравнение гиперповерхности $V_{2} \subset C \times \mathbb{P}, f^{(i)}$ - однородные многочлены степени $d \geqslant 3$ от координат $x_{*}$. Ряд

$$
f_{\xi}=\sum_{l=0}^{\infty} t^{l} f_{\xi}^{(l)}(x)=\sum_{l=0}^{\infty} t^{l} f^{(l)}\left(t^{w\left(x_{0}\right)} x_{0}, \ldots, t^{w\left(x_{M}\right)} x_{M}\right)
$$

обращается в нуль на $V_{1}$, а вне отмеченного слоя $F_{1}$, т.е. при $t \neq 0$, дает уравнение $V_{1}$. Пусть $b \in \mathbb{Z}_{+}-$максимальная степень параметра $t$, делящая $f_{\xi}$. Тогда

$$
t^{-b} f_{\xi}=g=\sum_{l=0}^{\infty} t^{l} g^{(l)}\left(x_{0}, \ldots, x_{M}\right)
$$

дает уравнение гиперповерхности $V_{1}$ и в отмеченном слое $X_{p}$.

Лемма 3. Для любого $l \in \mathbb{Z}_{+}$многочлен $f^{(l)}$ есть линейная комбинация мономов веса $\geqslant b-l$, а многочлен $g^{(l)}$ есть линейная комбиначия мономов вес $a \leqslant b+l$.

ДокАЗАТЕЛЬСТво. Предположим, что моном $x^{I}$ входит в многочлен $f^{(l)}$ с ненулевым коэффициентом. Тогда он порождает компоненту $t^{l+w\left(x^{I}\right)} x^{I}$ ряда $f_{\xi}$ и, более того, эта компонента порождается только этим мономом в $f^{(l)}$. Отсюда $l+w\left(x^{I}\right) \geqslant b$, как и утверждалось. Предположим, что моном $x^{I}$ входит в $g^{(l)}$ с ненулевым коэффициентом. Он происходит из монома $t^{l+b} x^{I}$ ряда $f_{\xi}$, который, в свою очередь, может происходить только из монома $x^{I}$ в составе многочлена $f^{\alpha}$, где $\alpha+w\left(x^{I}\right)=l+b$. Лемма доказана.

Пусть

$$
\begin{aligned}
& P_{*}=\left\{x_{j}=0 \mid w\left(x_{j}\right) \geqslant 1\right\}=\mathbb{P}\left\langle e_{j} \mid w\left(x_{j}\right)=0\right\rangle, \\
& P^{*}=\left\{x_{j}=0 \mid w\left(x_{j}\right) \leqslant m-1\right\}=\mathbb{P}\left\langle e_{j} \mid w\left(x_{j}\right)=m\right\rangle
\end{aligned}
$$

- подпространства минимального и максимального веса соответственно.

Лемма 4. Если $b \geqslant m+1$, mo $P_{*} \subset \operatorname{Sing} F_{2} . \operatorname{Ecлu} m(d-1) \geqslant b+1, m o$ $P^{*} \subset \operatorname{Sing} F_{1}$. 
ДоКАЗАТЕЛЬСТВо. Предположим, что $b \geqslant m+1$. Слой $F_{2} \subset \mathbb{P}$ над отмеченной точкой задается уравнением $f^{(0)}=0$. В силу предположения $f^{(0)}$ есть линейная комбинация мономов веса $\geqslant m+1$. Если моном $x^{I}$ входит в $f^{(0)}$ с ненулевым коэффициентом, то $x^{I}$ делится на квадратичный моном от переменных $\mathscr{A} \backslash \mathscr{A}_{*}$ (иначе $w\left(x^{I}\right) \leqslant m$ ). Поэтому все первые частные производные многочлена $f^{(0)}$ обращаются в нуль на $P_{*}$. Тем самым, $P \subset \operatorname{Sing} F_{2}$.

Аналогично, если $b \leqslant m(d-1)-1$, то любой моном $x^{I}$ в $g^{(0)}$ делится на квадратичный моном от $\mathscr{A} \backslash \mathscr{A}^{*}$, иначе имеем $w\left(x^{I}\right) \geqslant m(d-1)$, что противоречит предположению и лемме 3. Лемма 4 доказана.

Заметим теперь, что при $d \geqslant 3$ неравенства $b \leqslant m$ и $b \geqslant m(d-1)$ не могут выполняться одновременно. Следовательно, выполнено хотя бы одно из двух неравенств леммы 4 . Пусть $b \geqslant m+1$. Поскольку $V_{2}$ неособо, $P_{*}-$ точка. Пусть $\mathscr{A}_{*}=\left\{x_{0}\right\}$, так что $P_{*}=(1,0, \ldots, 0)$. Снова в силу того, что $V_{2}$ неособо, имеем $f^{(1)}(1,0, \ldots, 0) \neq 0$. Следовательно, моном $x_{0}^{d}$ входит в $f^{(1)}$ с ненулевьм коэффициентом. По лемме 3 получаем $b \leqslant 1$. Отсюда $m=0$ - противоречие. В случае $b \leqslant m(d-1)-1$ рассуждения симметричны: $V_{1}$ неособо, $P^{*}$ - точка $(0, \ldots, 0,1), \mathscr{A}^{*}=\left\{x_{M}\right\}$ и $g^{(1)}(0, \ldots, 0,1) \neq 0$, так что $m d \leqslant b+1$, откуда снова получаем $m=0$ - противоречие.

Значит, нетривиальных весов быть не может, и $\xi$ - послойньй бирегулярньй изоморфизм. Следовательно, и $\chi=\left.\xi\right|_{V_{1}}$ есть послойньй изоморфизм. Доказательство теоремы 1 закончено.

6. Расслоения на квадрики. Единственный момент, когда мы использовали условие $d \geqslant 3$, это получение одного из неравенств леммы 4 . Сами леммы 3,4 и заключительная часть доказательства теоремы остаются справедливыми и при $d=2$. Отсюда следует, что нетривиальное бирациональное преобразование при $d=2$ возможно только в случае $b=m$. Рассмотрим этот случай подробнее. Переменные веса нуль могут входить в $f^{(0)}$ только в мономах вида $c_{\alpha \beta} x_{\alpha} x_{\beta}, w\left(x_{\alpha}\right)=0, w\left(x_{\beta}\right)=m$. Пусть $L_{*}$ и $L^{*}-$ подпространства в $L$, натянутые на базисные векторы минимального и максимального веса соответственно, так что $P_{*}=\mathbb{P} L_{*}, P^{*}=\mathbb{P} L^{*}$. Пусть $q_{0}: L_{*} \times L^{*} \rightarrow \mathbb{C}$ - билинейная форма $\sum c_{\alpha \beta} x_{\alpha} x_{\beta}$. Если $q_{0}$ имеет нетривиальное ядро в $L_{*}$, то, как и в доказательстве теоремы $1, b=m \leqslant 1$. Это же верно, если $q_{0}$ имеет нетривиальное ядро в $L^{*}$. Следовательно, если $m \geqslant 2$, то $q_{0}-$ невырожденная билинейная форма, $\operatorname{dim} L_{*}=\operatorname{dim} L^{*}$ и заменой базиса в $L_{*}, L^{*}$ можно добиться того, что $q_{0}=\sum x_{i}^{(0)} x_{i}^{(m)}$ есть стандартная форма, $w\left(x_{i}^{(\alpha)}\right)=\alpha$. Рассуждая аналогичным образом, получаем следующий факт.

ТЕОрема 2. При $m=2 k$ четном координаты $\left(x_{*}\right)$ могут быть выбраны и затем перенумерованы в виде $x_{i}^{(j)}, w\left(x_{i}^{(j)}\right)=j$, таким образом, что $f^{(0)}=q+f_{+}^{(0)}$, где $f_{+}^{(0)}$ есть линейная комбинация мономов $x_{i}^{(\alpha)} x_{j}^{(\beta)}$ c $\alpha+\beta \geqslant m+1$, а q имеет вид

$$
q=\sum_{j=0}^{k-1} \sum_{i} x_{i}^{(j)} x_{i}^{(m-j)}+\sum_{i}^{\prime}\left(x_{i}^{(k)}\right)^{2}
$$

әде сумма $\sum^{\prime}$ распространяется либо на все переменные веса $k$ и тогда слои $F_{1}, F_{2}$ неособы, либо на все, кроме одной, $и$ тогда $F_{1}, F_{2}$ суть конусы с нульмерной верииной. Вершина конуса $F_{2}$ содержится в подпространстве $\mathbb{P} L_{\leqslant k}=$ $\mathbb{P}\left\langle e_{i}^{(j)}, j \leqslant k\right\rangle$, но не в $\mathbb{P} L_{\leqslant k-1}$. Наоборот, вериина конуса $F_{1}$ содержится в 
$\mathbb{P} L_{\geqslant k}=\mathbb{P}\left\langle e_{i}^{(j)}, j \geqslant k\right\rangle$, но не в $\mathbb{P} L_{\geqslant k+1}$. В любом случае $F_{1}$ задается уравнением $g^{(0)}=q+g_{-}^{(0)}$, где $g_{-}^{(0)}$ есть линейная комбиначия мономов $x_{i}^{(\alpha)} x_{j}^{(\beta)}$ с $\alpha+\beta \leqslant m-1$.

При $m=2 k+1$ нечетном координаты $\left(x_{*}\right)$ могут быть выбраны и перенумерованы в виде $x_{i}^{(j)}, w\left(x_{i}^{(j)}\right)=j$, таким образом, что $f^{(0)}=q+f_{+}^{(0)}, g^{(0)}=q+g_{-}^{(0)}$, где $f_{+}^{(0)}, g_{-}^{(0)}$ имеют тот же смысл, что и выие, а q имеет вид

$$
q=\sum_{j=0}^{k-1} \sum_{i} x_{i}^{(j)} x_{i}^{(m-j)}+\sum_{i} x_{i}^{(k)} x_{i}^{(k+1)}
$$

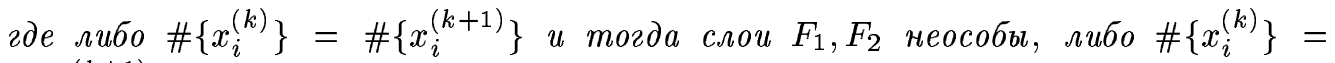
$\#\left\{x_{i}^{(k+1)}\right\} \pm 1$ и тогда слои $F_{1}, F_{2}$ суть конусы с нульмерной верииной.

Все эти возможности реализуются.

Таким образом, при $d=2$ наборы весов $\left\{a_{i}\right\}$ и размерности соответствуюших им подпространств не могут быть произвольными, а подчиняются соотношениям симметрии относительно половины максимального веса $m / 2$. Обратим внимание, что неособые слои перестраиваются в неособые, а особые- в особые, так что наличие или отсутствие особых точек на слое есть инвариант послойных бирациональных преобразований.

7. Описание структуры раздутий. Сохраняя обозначения п. 1 для диагонального представления (2) послойного отображения $\xi: X \rightarrow X$, положим $L_{a}=\left\langle e_{i}\right.$ | $\left.w\left(e_{i}\right)=a\right\rangle \subset L-$ подпространство, натянутое на базисные векторы веса $a$. Положим также

$$
L_{\leqslant a}=\bigoplus_{b \leqslant a} L_{b}, \quad L_{<a}=\bigoplus_{b<a} L_{b}, \quad L_{\geqslant a}=\bigoplus_{b \geqslant a} L_{b}, \quad L_{>a}=\bigoplus_{b>a} L_{b}
$$

и для каждого $? \in\{a, \leqslant a,<a, \geqslant a,>a\}$ положим $P_{?}=\mathbb{P} L_{\text {? }}$ и $R_{?}=P_{?} \times C$. Имеют место очевидные соотношения $L_{\leqslant a}=L_{<a} \oplus L_{a}, L=L_{\leqslant a} \oplus L_{>a}$ и т.д., и определены линейные проекции

$$
\begin{aligned}
& \pi_{\leqslant a}: \mathbb{P} \rightarrow P_{\leqslant a} \quad \text { из подпространства } P_{>a}, \\
& \pi_{<a}: \mathbb{P} \rightarrow P_{<a} \quad \text { из подпространства } P_{\geqslant a},
\end{aligned}
$$

так что, например, $\left.\pi_{<a}\right|_{P_{\leqslant}}: P_{\leqslant a} \rightarrow P_{<a}$ есть линейная проекция из подпространства $P_{a}$.

Пусть $\left\{a_{0}=0<a_{1}<\cdots<a_{k}\right\}$ - все веса диагонального представления (2). Положим $b_{i}=a_{i}-a_{i-1}$. Построим последовательность $b_{1}+\cdots+b_{k}=a_{k}=m$ раздутий $\varphi_{i, j}, i=1, \ldots, k, j=1, \ldots, b_{i}$, следуюшим образом (см. диаграмму ниже). Раздутия $\varphi_{i, j}$ имеют вид

$$
\begin{array}{rcc}
\varphi_{i, j}: X_{i, j} & \longrightarrow & X_{i, j-1} \\
\cup & & \cup \\
E_{i, j} & \longrightarrow & B_{i, j-1}
\end{array}
$$

где $j \geqslant 1, B_{i, j-1}-$ гладкий центр раздутия, $E_{i, j}-$ исключительный дивизор. Собственньй прообраз цикла $Y$ на $X_{i, j}$ обозначается добавлением верхнего индекса $i, j: Y^{i, j}$. 
Первьй раздуваемьй цикл есть $B_{1,0}=P_{\geqslant a_{1}}=P_{\geqslant 1}$;

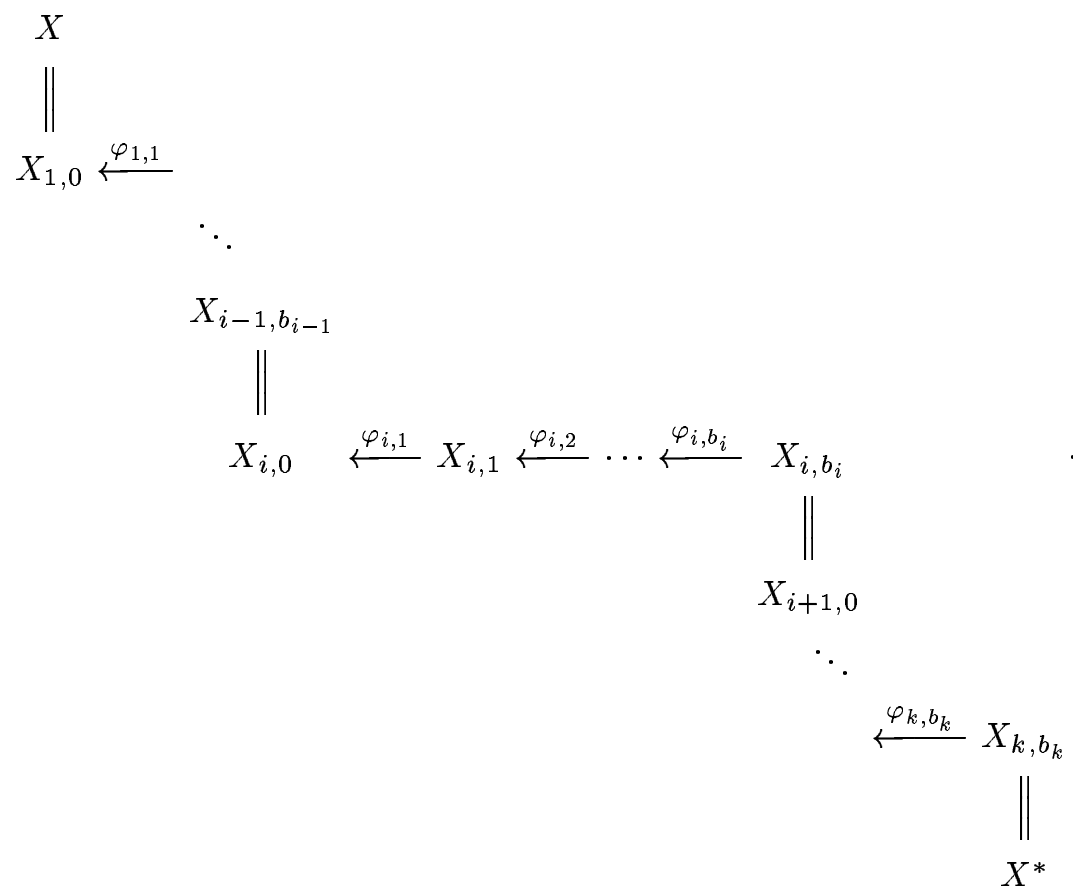

Для $1 \leqslant j \leqslant b_{i}$ имеем $B_{i, j-1}=E_{i, j-1} \cap R_{\geqslant a_{i}}^{i, j-1} \cong P_{\geqslant a_{i}}$ (последний изоморфизм легко доказьвается по индукции), так что $\varphi_{i, j}: E_{i, j} \rightarrow B_{i, j-1} \cong P_{\geqslant} a_{i}$ есть $\mathbb{P}^{c}(i)$-расслоение над $P_{\geqslant a_{i}}$, где $c(i)=\operatorname{codim}_{\mathbb{P}} P_{\geqslant a_{i}}=\operatorname{dim} P_{<a_{i}}+1$.

Для упрощения обозначений положим: $E_{i, j}^{+}-$собственньй прообраз $E_{i, j}$ на следующем раздутии, $(i, j)_{+}-$следующий за $(i, j)$, a $(i, j)_{-}-$предшествуюший $(i, j)$ бииндексы соответственно.

Легко видеть, что $E_{i, j}^{+} \cap B_{(i, j)_{+}}=\varnothing$, так что $E_{i, j}^{+} \cong E_{i, j}^{k, b_{k}}$. Положим $S_{i, j}=E_{i, j} \cap$ $R_{\geqslant a_{i}}^{i, j} \cong P_{\geqslant a_{i}}$ при $0 \leqslant j \leqslant b_{i}$, так что для $j=0, \ldots, b_{i}-1$ имеем $S_{i, j}=B_{i, j}$. Для $j \stackrel{1}{=} 1, \ldots, b_{i}$ цикл $S_{i, j}$ есть сечение расслоения $\varphi_{i, j}: E_{i, j} \rightarrow B_{i, j-1}$. Имеет место диаграмма

$$
\begin{gathered}
S_{i+1,0}=B_{i+1,0} \cong P_{\geqslant a_{i+1}}=P_{>a_{i}} \\
\cap, \\
S_{i, b_{i}} \cong P_{\geqslant a_{i}}
\end{gathered},
$$

где горизонтальные изоморфизмы естественны в том смысле, что раздутия $\varphi_{\alpha, \beta}$ с $\alpha \leqslant i$ не меняют подмногообразий $R_{\geqslant a_{i}}$ : на $R_{\geqslant a_{i}}$ раздувается дивизор. В частности, при $2 \leqslant j \leqslant b_{i}$ цикл $B_{i, j-1} \subset E_{i, j-1}$ есть сечение расслоения $\varphi_{i, j-1}: E_{i, j-1} \rightarrow B_{i, j-2}$. Отметим, что $E_{(i, j)_{-}}^{+} \cap S_{i, j}=\varnothing$. Положим $\Gamma_{i, j}=E_{(i, j)_{-}}^{+} \cap E_{i, j}$. В силу только что сказанного $\Gamma_{i, j} \cap S_{i, j}=\varnothing$. Для удобства обозначим через $E_{1,0}$ слой $X_{p}$ над отмеченной точкой $p \cong \mathbb{P}$.

ПРЕДЛОЖЕНИЕ 1. Описанная последовательность раздутий разрешает особенности $\xi$, т.е. $\xi: X^{*} \rightarrow X$ продолжается до (бирационального) морфизма $\bar{\xi}$. 
ДокАЗАТЕльСтво - прямые вычисления в локальных координатах.

8. Стягивания. Опишем теперь действие морфизма $\bar{\xi}: \cup E_{i, j}^{+} \rightarrow \mathbb{P}$. Прежде всего, $E_{1,0} \cong \mathbb{P}, E_{1,0}^{+}$есть раздутие $\mathbb{P}$ вдоль $P_{\geqslant a_{1}}$ и $\left.\bar{\xi}\right|_{E_{1,0}^{+}}$есть регулярное продолжение линейной проекции $\mathbb{P} \rightarrow P_{0}$ из $P_{\geqslant} a_{1}$.

Морфизмы

$$
\xi_{i, j}=\left.\bar{\xi}\right|_{E_{i, j}^{+}}: E_{i, j}^{+} \rightarrow \mathbb{P}
$$

опишем по индукции. Пусть $1 \leqslant j \leqslant b_{i}-1$ и $\xi_{\alpha, \beta}$ для предшествуюших $(\alpha, \beta)$ уже построены. Легко проверить, что

$$
E_{i, j} \supset \Gamma_{i, j} \cong P_{\geqslant a_{i}} \times P_{<a_{i}}
$$

Напомним, что $\Gamma_{i, j}$ содержится в $E_{(i, j)_{-}}^{+}$, так что $\left.\bar{\xi}\right|_{\Gamma_{i, j}}=\left.\xi_{i, j-1}\right|_{\Gamma_{i, j}}$ уже построено. На самом деле $\left.\bar{\xi}\right|_{\Gamma_{i, j}}$ есть просто проекция на $P_{<a_{i}}$. Далее, послойная проекция $\gamma_{i, j}: E_{i, j} \rightarrow \Gamma_{i, j}$ из сечения $S_{i, j} \subset E_{i, j}$ регуляризуется до морфизма $\gamma_{i, j}^{+}: E_{i, j}^{+} \rightarrow \Gamma_{i, j}$ (в слое расслоения $\varphi_{i, j}: E_{i, j} \rightarrow B_{i, j-1}$ над точкой $x \in B_{i, j-1}$ проекция $\gamma_{i, j}$ есть проекция из точки $S_{i, j} \cap \varphi_{i, j}^{-1}(x)$ на гиперплоскость $\Gamma_{i, j} \cap \varphi_{i, j}^{-1}(x)$ в этом слое). Теперь

$$
\xi_{i, j}=\left(\left.\xi_{i, j}\right|_{\Gamma_{i, j}}\right) \circ \gamma_{i, j}^{+}: E_{i, j}^{+} \rightarrow P_{<a_{i}} .
$$

Осталось описать морфизм $\xi_{i+1,0}: E_{i+1,0}^{+}=E_{i, b_{i}}^{+} \rightarrow \mathbb{P}$. Для упрощения обозначений мы будем опускать индексы и писать $\xi$ вместо $\xi_{i+1,0}, E$ вместо $E_{i+1,0}, E^{+}$вместо $E_{i+1,0}^{+}, S$ вместо $S_{i+1,0}, \Gamma$ вместо $\Gamma_{i+1,0}, a$ вместо $a_{i}, \Gamma^{+}$вместо $\Gamma_{i+1,1}, \varphi$ вместо $\varphi_{i, b_{i}}, \varphi^{+}$вместо $\varphi_{i+1,1}, B_{-}$вместо $B_{i, b_{i}-1}, B$ вместо $B_{i+1,0}$.

Итак, $S \cong P_{\geqslant a}$ - сечение расслоения $\varphi: E \rightarrow B_{-}$. Пусть $S^{+} \subset E^{+}-$собственньй прообраз $S$ на $E^{+}$. Геометрически $S^{+}$есть раздутие $P_{\geqslant a}$ вдоль $P_{>a}$. Теперь $\xi: S^{+} \rightarrow P_{a}$ есть регулярное продолжение линейной проекции $P_{\geqslant a} \rightarrow P_{a}$ из подпространства $P_{>a}$.

Далее, $\Gamma \cong P_{\geqslant a} \times P_{<a}$. Отображение $\left.\xi\right|_{\Gamma}$ построено на предыдущем шаге. Легко проверить, что $\left.\xi\right|_{\Gamma}$ есть проекция на второй сомножитель $P_{<a}$.

Пусть $x \in B_{-} \backslash \varphi(B)$. Слой расслоения $\varphi$ над $x$ обозначим через $F(x)$. На нем имеется точка $S(x)=S \cap F(x)$ и гиперплоскость $\Gamma(x)=\Gamma \cap F(x)$. Значение $\xi(S(x))=s(x) \in P_{a}$ мы уже знаем. Изоморфизм $\xi: \Gamma(x) \rightarrow P_{<a}$ уже построен. Теперь $\left.\xi\right|_{F(x)}$ есть изоморфизм $F(x)$ и линейного пространства $\left\langle P_{<a}, s(x)\right\rangle$.

Наконец, пусть $x \in \varphi(B)$. Опишем действие $\xi$ на слое морфизма $\varphi \circ \varphi^{+}: E^{+} \rightarrow B_{-}$ над точкой $x$. Этот слой приводим и имеет две компоненты $F^{+}(x) \cup \Gamma^{+}(x)$, где $F^{+}(x)-$ собственньй прообраз слоя $\varphi^{-1}(x)$ на $E^{+}$.

Имеем $\Gamma^{+} \cong P_{>a} \times P_{\leqslant a}$ и $\Gamma^{+}(x) \cong P_{\leqslant a}-$ слой проекции на первьй сомножитель. Морфизм $\left.\xi\right|_{\Gamma^{+}}$есть проекция на второй сомножитель $P_{\leqslant a}$, так что $\xi: \Gamma^{+}(x) \rightarrow P_{\leqslant a}$ есть изоморфизм проективных пространств.

Цикл $F^{+}(x)$ есть проективное пространство размерности $\operatorname{dim} P_{<a}+1$ с раздутой точкой $S(x)$. Гиперплоскость $\Gamma(x) \subset F^{+}(x)$ канонически изоморфна $P_{<a}$, причем $S(x) \notin \Gamma(x)$. Теперь $\left.\xi\right|_{F+(x)}$ есть регуляризация линейной проекции из точки $S(x)$ на $P_{<a} \cong \Gamma(x)$.

Отметим, что все дивизоры $E_{i, j},(i, j) \neq\left(k, b_{k}\right)$, теряют размерность на $\mathbb{P}$ и, кроме того, $\bar{\xi}\left(E_{k, b_{k}}\right)=\mathbb{P}$. 
9. Пример. Пусть $V \in \mathscr{V}(2)$ - расслоение на квадрики, $F_{p} \subset \mathbb{P}$ - слой над отмеченной точкой. Предположим, что $M=2 k+1$ нечетно и слой $F_{p}$ неособ. При $m=1$ перестройка многообразия $V$ есть раздутие плоскости $P \subset F_{p}$ максимальной размерности $(M-1) / 2$ и последующее стягивание раздутого проективного пространства $X_{p}$ на другую плоскость $Q \subset X_{p}$ размерности $(M-1) / 2$. Например, при $M=3$ берем прямую $P \subset F_{p}$ на $F_{p}$, раздуваем $P$ и затем стягиваем собственньй прообраз $\widetilde{F}_{p} \cong F_{p}$ на прямую $Q: F_{p} \cong Q \times \mathbb{P}^{1}$. В связи с этим возникает вопрос: можно ли разложить любую перестройку многообразия $V$ в композицию элементарньх перестроек $\varphi_{P, Q}$ для всех пар плоскостей максимальной размерности? И еще вопрос: какова группа бирациональных автоморфизмов $V$, индуцирующих бирегулярный автоморфизм на слое общего положения? В последнем случае речь идет об описании вырождений одномерных семейств проективных изоморфизмов $\xi_{x} \in \mathrm{Aut} \mathbb{P}$, сохраняюших квадрику $F_{x} \subset V$.

\section{СПИСОК ЦИТИРОВАННОЙ ЛИТЕРАТУРЫ}

[1] Исковских В. А., Манин Ю. И. Трехмерные квартики и контрпримерык проблеме Люрота // Матем. сб. 1971. Т. 86. №1. С. 140-166.

[2] Саркисов В.Г. О структурах расслоений на коники // Изв. АН СССР. Сер. матем. 1982. T. 46. № 2. C. 371-408.

[3] Пухликов А.В. Бирациональные автоморфизмы трехмерных алгебраических многообразий с пучком поверхностей Дель Пеццо // Изв. РАН. Сер. матем. 1998. Т. 62. № 1. С. 123-164.

[4] Pukhlikov A. V. Birational automorphisms of Fano hypersurfaces // Invent. Math. 1998. V. 134. № 2. P. 401-426.

[5] Пухликов А. В. Бирационально жесткие расслоения Фано // Изв. РАН. Сер. матем. 2000. T. 64 . № 3 .

[6] Гриненко М. М.Бирациональные свойства пучков поверхностей дель Пеццо степени 1 и 2 // Матем. сб. 2000. Т. 191. № 5. С. 17-38.

[7] Corti A. Del Pezzo surfaces over Dedekind schemes // Ann. of Math. 1996. V. 144. P. 641-683.

[8] Park J. Birational maps of del Pezzo fibrations. Preprint. Baltimore: Johns Hopkins Univ., 1999.

[9] Cheltsov I. A., Park J. Eckard points on quartic 3-fold. Preprint. Baltimore: Johns Hopkins Univ., 2000.

[10] Шокуров В. В. Трехмерные лог-перестройки // Изв. АН СССР. Сер. матем. 1992. Т. 56. № 1. C. $105-203$.

[11] Kollár J. Flips and Abundance for Algebraic Threefolds. Astérisque. V. 211, 1993.

[12] Iskovskikh V. A., Pukhlikov A. V. Birational automorphisms of multi-dimensional algebraic varieties // J. Math. Sci. 1996. V. 82. P. 3528-3613.

Математический институт им. В. А. Стеклова РАН

Поступило

E-mail: pukh@mi.ras.ru

22.02 .2000 